Artículos de Revisión

\title{
La genética como factor pronóstico y terapéutico en el mieloma múltiple
}

\author{
G uillermo Conte $L^{1,4}$, Esteban Braggio², G astón Figueroa ${ }^{1}$, \\ Rafael Fonseca ${ }^{3}$.

\section{Genetic markers as prognostic factors in multiple myeloma}

The search for prognostic factors in multiple myeloma has identified the genetic profile of the tumor as the main determinant of patient survival and response to treatment. There is an association between a dismal prognosis and the presence of $t(4: 14)$ translocations or 17p deletion, determined by fluorescent in situ hybridization (FISH) or the detection of chromosome 13 deletion using conventional cytogenetic techniques. These alterations define a subpopulation that comprises $25 \%$ of patients with a bad prognosis even if they are treated with high dose chemotherapy. These patients should be early derived to more specific therapies. In the other hand, the other $75 \%$ of patients without a genetic risk factor, have a higher probability of success with conventional treatment (Rev Méd Chile 2009; 137: 552-8).

(Key words: Citogenetics; Genetic markers; Multiple myeloma)

Recibido el 23 de noviembre, 2007. Aceptado el 7 de julio, 2008.

${ }^{1}$ Sección de Hematología, Hospital Clínico Universidad de Chile, Santiago de Chile. ${ }^{2}$ Mayo Clinic Scottsdale, Collaborative Research Building, Scottsdale, Arizona, USA. ${ }^{3}$ Division of Hematology and Oncology, Mayo Clinic Comprehensive Cancer Center, Scottsdale, Arizona, ${ }^{4}$ International Myeloma Foundation Latin America.

$\mathrm{E}$ mieloma múltiple (MM) es una neoplasia de células B caracterizada por la proliferación clonal de células plasmáticas en la médula ósea y la presencia de una paraproteína en el suero $u$ orina. Sus principales manifestaciones clínicas son anemia, insuficiencia renal, hipercalcemia y lesiones osteolíticas ${ }^{1}$. La incidencia reportada internacionalmente varía de 0,2 a 5,1 casos x 100.000 habitantes-año ${ }^{2}$. En Chile se estima una incidencia de 2,2 casos por 100.000 habitantes, lo que representa unos 330 casos nuevos al año ${ }^{3}$.

Correspondencia a: Dr. Guillermo Conte L. Sección de Hematología, Hospital Clínico Universidad de Chile, Santos Dumont 999, Independencia, Santiago, Chile. Fax: 7777618. E mail: gfconte@gmail.com
El MM tiene una fase premaligna que incluye la gamopatía monoclonal de significado indeterminado (GMSI) y el mieloma múltiple asintomático (MMA). Los criterios diagnósticos de GMSI, MM asintomático y MM sintomático han sido recientemente actualizados y se presentan en la Tabla $1^{4}$. La diferenciación en etapas de progresión es crítica para poder distinguir el MM activo de las fases que lo preceden, ya que el tratamiento usualmente no se inicia hasta que no sea evidente la sintomatología relacionada con el MM. Los estudios clínicos realizados no han conseguido demostrar ningún tipo de ventaja cuando el tratamiento es iniciado en las etapas más precoces de la enfermedad. Si a lo anterior se le suma la falta de una estrategia curativa, iniciar el tratamiento hasta la aparición de los síntomas clínicos continúa siendo un abordaje razonable ${ }^{5,6}$. 


\section{Tabla 1. C riterios diagnóstico de G M SI, M M asintomático y M M sintomático - International M yeloma Working Group ${ }^{4}$}

Gamopatía Monoclonal de Significado Indeterminado (GMSI)

Se requieren los 3 criterios:

1. Paraproteína monoclonal en el suero $<3 \mathrm{gr} / \mathrm{dL}$ o en la orina $<1 \mathrm{gr} / 24 \mathrm{hr}$.

2. Plamocitosis en médula ósea inferior a $10 \%$.

3. Calcio, hemoglobina y creatinina normales.

Ausencia de lesiones óseas, ausencia de datos clínicos de amiloidosis.

Mieloma Múltiple Asintomático

Se requieren los 2 criterios:

1. Paraproteína monoclonal en el suero $>3 \mathrm{gr} / \mathrm{dL}$ o infiltración plasmática de la médula ósea $>10 \%$.

2. Ausencia de síntomas o evidencias de disfunción orgánica en relación con el mieloma (CRAB)

Mieloma Múltiple Sintomático

Se requieren los 3 criterios:

1. Plamocitosis monoclonal en médula ósea o plasmocitoma.

2. Presencia de paraproteína monoclonal en el suero 0 en la orina.

3. Disfunción orgánica en relación con el mieloma (1 o más criterios)

C\} Elevación del calcio $>0,5 \mathrm{mmol} / \mathrm{L}(1 \mathrm{mg} / \mathrm{dL})$ sobre el límite normal o $>2,75 \mathrm{mmol} / \mathrm{L}(11 \mathrm{mg} / \mathrm{dL})$.

R\} Insuficiencia renal: Creatininemia $>173 \mathrm{mmol} / \mathrm{L}(1,96 \mathrm{mg} / \mathrm{dL})$.

A\} Anemia: Hemoglobina $<10 \mathrm{gr} / \mathrm{dl}$ o $2 \mathrm{gr} / \mathrm{dl}$ por debajo de la normalidad.

B \} Lesiones líticas u osteoporosis con fracturas de compresión.

Otros: Hiperviscosidad sintomática, amiloidosis, infecciones bacterianas recurrentes ( $>2$ episodios en 12 meses).

El pronóstico de los pacientes con MM es muy variable, con una mediana de sobrevida de 2,5 a 3 años ${ }^{7}$. En una serie de 245 pacientes tratados en 6 centros nacionales la sobrevida a 5 años fue de solamente $23 \%{ }^{8}$. A pesar de la aparición de importantes avances terapéuticos ${ }^{9,10}$, la enfermedad continúa siendo incurable y hay una importante proporción de pacientes $(10 \%-20 \%)$ que fallecen precozmente luego del diagnóstico ${ }^{11,12}$.

Durante las últimas décadas se han logrado importantes avances en la identificación de factores pronósticos. Entre éstos, aparece el perfil genético del tumor como el más importante determinante de la sobrevida de los pacientes y de la respuesta al tratamiento ${ }^{13,14}$. En las próximas páginas describiremos el conocimiento actual sobre la genética del mieloma y comentaremos los primeros intentos en realizar terapias individualizadas basándose en el conocimiento de las alteraciones genéticas.

\section{GeNÉTICA DEL MIELOMA MÚLTIPLE}

El MM es un tumor maligno de células B de origen post-centro germinal. Las células neoplásicas se caracterizan por presentar mutaciones en las regiones variables de las cadenas pesadas $(\mathrm{IgH})$ y livianas (IgL) de inmunoglobulinas, consecuencia de los procesos de hipermutación somática y selección antigénica. La mayoría de los tumores presentan el switch de isotipo de la cadena de $\mathrm{IgH}$ y expresan con mayor frecuencia IgG e IgA y raramente IgD e IgE. Muy infrecuentemente expresan sólo IgM (1\%) y hasta $15 \%$ de los casos expresan sólo cadenas livianas ${ }^{15}$.

Una de las principales características genéticas de $\mathrm{MM}$ es la presencia de translocaciones que involucran el locus de IgH (14q32) o uno de los loci de IgL (Igk 2p12 e Igd 22q11) ${ }^{16}$. Se piensa que estas translocaciones resultan de errores en uno de los tres procesos específicos de las células B que

ARtículo

DE REVISIÓN 
modifican el ADN: (1) recombinación VDJ; (2) hipermutación somática y (3) switch de isotipo de IgH. La consecuencia de estas translocaciones es la desregulación o aumento en la expresión de un oncogén que se posiciona cerca de uno o más segmentos reguladores (enhancers) de los genes de $\operatorname{Ig}^{17}$.

Un evento inicial en el origen de muchos casos de MM es la translocación cromosómica que involucra las regiones switch del gen de $\mathrm{IgH}$ (14q32) y varias otras regiones no aleatorias, en las cuales se hayan localizados los genes de la familia de las ciclinas D (ciclina D3 6p21, ciclina D1 11q13), los miembros de la familia MAF (cMAF 16q23, MAFB 20q12) y el receptor 3 del factor de crecimiento de los fibroblastos (FGFR3 4p16) (13-14) ${ }^{16}$. Estas translocaciones recurrentes han sido identificadas en aproximadamente 50\% de las muestras primarias de pacientes con MM. La otra mitad de los pacientes se caracteriza por la presencia de múltiples trisomías (hiperdiploidia), más comúnmente de los cromosomas $3,5,7,9$, $11,15,19,21^{16,18}$. La evolución oncogénica del grupo hiperdiploide es menos comprendida ${ }^{18}$. Estos eventos genéticos primarios o tempranos (translocaciones de IgH e hiperdiploidia) presentan en común la sobreexpresión de una o más ciclinas D (D1-D3) en la casi totalidad de los pacientes con MM por lo que se ha propuesto que la sobreexpresión de ciclina D es un evento clave en la patogenia del $\mathrm{MM}^{19}$.

Luego de estas alteraciones genéticas primarias la progresión de la enfermedad se caracteriza por la ocurrencia de eventos secundarios (translocaciones, deleciones, mutaciones) consecuencia de la inestabilidad genómica de las células neoplásicas $^{17}$. Entre éstas destacan la monosomía/deleción del cromosoma 13, la deleción del cromosoma 17 y la amplificación del cromosoma $1^{16,20}$, todas con importantes implicancias pronósticas. Otras alteraciones genéticas comunes son las mutaciones somáticas en genes tales como P53 (con la consiguiente pérdida de función), FGFR3, NRAS y KRAS (produciendo la sobreactivación en los 3 casos) u otras translocaciones secundarias que se originan por medio de mecanismos no involucrados en la diferenciación de las células $\mathrm{B}^{16}$. Una región que comúnmente se encuentra presente en las translocaciones secundarias es la 8q24, donde está localizado el gen $\mathrm{MYC}^{21}$.

\section{IMPLCACIONES CLÍNICAS DE LAS ALTERACIONES GENÉTICAS EN EL MM}

Prácticamente en todos los casos de MM es posible identificar anormalidades citogenéticas cuando son estudiados por FISH (fluorescent in situ hybridization). Por el contrario utilizando citogenética convencional solamente un tercio de los casos tiene un cariotipo anormal, el que usualmente es muy complejo, alcanzando un promedio de 11 anormalidades cromosómicas ${ }^{22}$. Utilizando FISH es posible agrupar los cariotipos en dos grupos: hiperdiploide y no-hiperdiploi$\mathrm{de}^{15}$. El subtipo hiperdiploide se caracteriza por la presencia de múltiples trisomías (más comúnmente los cromosomas $3,5,7,9,11,15,19,21)$ y una baja frecuencia de translocaciones que incluyan IgH. El subtipo no-hiperdiploide se caracteriza por una alta frecuencia de translocaciones IgH y una frecuente pérdida de cromosomas, especialmente los cromosomas 13, 14, 16 y 18.

Hiperdiploidia. La presencia de la hiperdiploidia es considerada generalmente un evento favorable. Hasta el momento, todos los estudios realizados han demostrado una mayor sobrevida global y libre de progresión en los pacientes que presentan hiperdiploidia, detectada mediante la determinación del contenido de ADN por citometría de flujo o por análisis cariotípico ${ }^{13,23,24}$. Esta diferencia en la sobrevida no se refleja en el tipo de respuesta inicial al tratamiento utilizando estrategias basadas en el uso del melfalán, sino que se manifiesta en una duración más prolongada de la remisión ${ }^{25}$. La sobrevida más prolongada del grupo hiperdiploide no puede ser explicada por los factores pronósticos clásicos (albúmina, proteína $\mathrm{C}$ reactiva, beta-2-micloglobulina), ya que éstos no demuestran diferencias significativas cuando son comparados con el grupo de pacientes no hiperdiploide. Toda esta información plantea que el principal factor relacionado con la diferente sobrevida del grupo de MM hiperdiploide sea intrínseco a sus características genéticas ${ }^{18}$.

$\mathrm{t}(11 ; 14)(\mathrm{q} 13 ; \mathrm{q} 32)$. La $\mathrm{t}(11 ; 14)(\mathrm{q} 13 ; \mathrm{q} 32)$ se detecta por cariotipo de metafase en $5 \%$ y por FISH en $15 \%-20 \%$ de los pacientes con $\mathrm{MM}^{16}$. Esta translocación produce la sobreexpresión del gen de ciclina D1 y este subgrupo de pacientes tienen 
con frecuencia un mieloma oligosecretor o de cadenas livianas, expresan CD20 y morfología linfoplasmocitaria 26 . Aunque inicialmente la presencia de $\mathrm{t}(11 ; 14)(\mathrm{q} 13 ; \mathrm{q} 32)$ caracterizaba un subgrupo de pacientes con mejor pronóstico, estudios recientes en pacientes tratados con quimioterapia en altas dosis y trasplante autólogo de médula ósea (TAMO) sugieren un efecto neutro 24,27 .

$\mathrm{t}(4 ; 14)(\mathrm{p} 16.3 ; \mathrm{q} 32)$. Esta translocación, críptica para la citogenética convencional, se detecta por FISH en $15 \%$ de los pacientes con MM. Genéticamente se caracteriza por la formación de un gen de fusión IgH-MMSET y sobreexpresión de FGFR3 ${ }^{28}$. Por análisis de expresión génica global se ha detectado cambios en la expresión de 127 genes ${ }^{29}$. Varios estudios han demostrado que la translocación $\mathrm{t}(4 ; 14)$ está asociada con una sobrevida menor en los pacientes tratados con quimioterapia convencional o TAMO 24,27,30,31. Un estudio reciente investigó la frecuencia de la $\mathrm{t}(4 ; 14)$ y su relevancia en el pronóstico en un grupo de 238 pacientes con MM que recibieron melfalán en altas dosis $\left(200 \mathrm{mg} / \mathrm{m}^{2}\right)$ seguido de trasplante autólogo de médula ósea ${ }^{24}$. La t $(4 ; 14)$ fue detectada en 26 de 153 pacientes (17\%). El grupo de pacientes con la translocación presentó una menor sobrevida libre de eventos post-TAMO (mediana 8,2 vs 17,8 meses; $\mathrm{p}<0,001$ ) y una sobrevida global significativamente menor (mediana 18,8 vs 43,9 meses; $p<0,001$ ) que los pacientes sin la translocación.

$t(14 ; 16)(q 32 ; q 23)$. La $t(14 ; 16)$ es detectada en $2 \%$ a $10 \%$ de los pacientes y en cerca de $25 \%$ de las líneas celulares de $\mathrm{MM}^{16}$. Como resultado de la translocación, el factor de transcripción c-maf es sobremegulado a nivel transcripcional ${ }^{32}$. La sobreexpresión de c-maf estimula la progresión en el ciclo celular mediante la sobreexpresión de ciclina D2 y promueve la interacción con el microambiente de la médula ósea vía la sobreexpresión de la integrina ß7, interacción que aumenta significativamente la secreción de VEGF, fenómenos importantes en la sobrevida de la célula mielomatosa ${ }^{33}$. En una serie se demostró la asociación entre la presencia de la $\mathrm{t}(14 ; 16)$ (q32;q23) y una sobrevida menor en pacientes tratados con quimioterapia convencional ${ }^{23}$. d13q14. La deleción 13q14/monosomía del cromosoma 13 es detectada por citogenética en $10 \%$ a $20 \%$ de los casos de MM, mientras que con FISH es posible identificarlas en $30 \%$ a $55 \%$ de los casos en todas las fases del mieloma, incluso en la GMSI y en el $\mathrm{MMA}^{16}$. Diversos estudios han reportado una fuerte asociación entre deleción del 13q identificada por citogenética convencional con una menor tasa de respuestas, resistencia a drogas y corta sobreviva $25,34,35$. Por el contrario las anormalidades detectadas por FISH no han sido claramente asociadas a mal pronóstico ${ }^{35}$, la razón de esta diferencia no ha sido aclarada. Estudios de análisis de expresión génica global han demostrado una sobreexpresión de genes relacionados al control del ciclo celular en pacientes con esta deleción, explicando quizás el peor pronóstico asociado $^{36}$.

Deleción de 17p13 (p53). La deleción de la región 17 p13 está presente en $10 \%$ de los pacientes con MM y está asociada con una sobrevida menor en pacientes tratados con quimioterapia convencio$\mathrm{nal}^{37}$. Datos recientes obtenidos de varias series de pacientes tratados con TAMO han confirmado el pronóstico muy desfavorable de los pacientes con la deleción de p5324,27.

Anormalidades del cromosoma 1. Las anormalidades del cromosoma 1 son una de las alteraciones estructurales más frecuentes en el MM ${ }^{20,38}$ y solo recientemente se ha podido identificar un gen relacionado con estas alteraciones. En recientes estudios se ha correlacionado la amplificación de la región cromosómica 1q21 con la sobreexpresión del gen CKS1B. El producto de este gen forma parte de un complejo sistema que regula la entrada a la fase S del ciclo celular ${ }^{39}$. En estudios con pacientes tratados con TAMO la sobreexpresión del gen CKS1B se ha asociado a menor sobrevida ${ }^{40,41}$.

Mutaciones Ras. Mutaciones activadoras de Ras han sido detectadas en 35\% a 50\% de los pacientes ${ }^{42,43}$. La frecuencia de las mutaciones puede aumentar en las fases más avanzadas de la enfermedad. Mutaciones en K-ras, pero no en $\mathrm{N}$ ras, han sido asociadas con una sobrevida menor ${ }^{42}$. 


\section{VALOR CLÍNICO DE LOS TESTS GENÉTICOS Y SUS IMPLICANCIAS TERAPÉUTICAS}

Existen informaciones suficientes para que se recomiende la adopción de tests genético-moleculares en la rutina del estudio de los pacientes con MM. Sin embargo, se requerirá que estas técnicas citogenéticas y de perfiles de expresión génica sean suficientemente validadas y consensuadas entre los diferentes centros de investigación para que puedan ser incorporadas en el manejo clínico habitual. Estudios independientes han identificado una asociación entre un pronóstico desfavorable y la presencia de translocaciones que involucran la cadena pesada de las inmunoglobulinas, con la excepción de $(11 ; 14)$ que parece tener un pronóstico neutro $24,27,31,35$. La detección de las $t(4 ; 14)$, t $(14,16)$ o de la deleción de p53 por FISH, así como la detección de la deleción del cromosoma 13 por citogenética define una subpoblación constituida por $25 \%$ de pacientes que tienen un pronóstico desfavorable aun si son tratados con TAMO y que deberían ser guiados precozmente hacia terapias más dirigidas. Por otro lado, el restante $75 \%$ de pacientes que carece de los factores de riesgo más desfavorables tiene una mayor probabilidad de beneficiarse con el TAMO

Estas consideraciones han sido la base de la propuesta del grupo de expertos de la Clínica Mayo para un abordaje terapéutico del MM basado en grupos de riesgo definidos por características genéticas (Mayo Stratification of Myeloma and Risk-adapted Therapy o mSMART, Tabla 2$)^{44}$. Bajo esta perspectiva, los pacientes de alto riesgo debieran ser enrolados en estudios clínicos que evalúen combinaciones de nuevos medicamentos que han demostrado alta actividad en mieloma múltiple (ej. bortezomib, lenalidomida, doxorrubicina liposomal) tanto en la fase de inducción como de mantención. Es así como recientes estudios avalan esta estrategia terapéutica. En un análisis de pacientes con $\mathrm{MM}$ en recaída 0 refractario tratados con bortezomib, la respuesta fue similar en los pacientes con o sin del(13), lo que sugiere que esta droga puede contrarrestar el efecto negativo de esta deleción ${ }^{45}$. Otro estudio que evaluó la respuesta a un esquema de inducción con bortezomib y dexametasona antes del TAMO, la presencia de $\operatorname{del}(13)$ o $t(4 ; 14)$ no modificaron la respuesta ${ }^{46}$. En una serie de pacientes no candidatos a TAMO tratados con melfalán, prednisona y lenalidomida, la sobrevida libre de enfermedad fue similar en los pacientes con $0 \sin t(4 ; 14)^{47}$.

\section{CONCLUSIONES}

El los últimos años se han logrado muy importantes avances en el tratamiento de los pacientes con MM, lográndose tasas de respuesta nunca antes vistas. Este creciente arsenal terapéutico nos obliga a definir de la mejor forma posible el pronóstico de los pacientes. El detallado conocimiento de la biología del mieloma nos ha permitido identificar a la genética de las células neoplásicas como el principal determinante de la respuesta al tratamiento y la sobrevida de los pacientes. Por esto se ha propuesto un abordaje terapéutico basado en la clasificación de grupos pronósticos determina-

Tabla 2. Clasificación de M M basado en grupos de riesgo definidos por características genéticas (mSM ART ) ${ }^{44}$

\begin{tabular}{|ll|}
\hline Alto riesgo ( 25\% de los pacientes) & Riesgo estándar ( 75\% de los pacientes) \\
\hline Presencia de cualquiera de lo siguiente: & Todas las otras anormalidades identificadas \\
& por FISH o citogenética, incluyendo: \\
- Del(17p) x FISH & - Hipendiploidía \\
- Translocación $(4 ; 14) \times$ FISH & Translocación $(11 ; 14) \times$ FISH \\
- Translocación $(14 ; 16) \times$ FISH & Translocación $(6 ; 14) \times$ FISH \\
- Hel(13q) x citogenética & \\
- PCLI $\geq 3 \%$ & \\
\hline
\end{tabular}


dos por características genéticas. Los pacientes en que al momento del diagnóstico se identifiquen características genéticas de alto riesgo pueden ser rápidamente enrolados en estudios clínicos o ser tratados con esquemas terapéuticos que incluyan

\section{REFERENCIAS}

1. Kyle Ra, Rajkumar SV. Multiple Myeloma. N Engl J Med 2004; 351: 1860-73.

2. SiROHI B, Powles R. Epidemiology and outcomes research for MGUS, myeloma and amyloidosis. Eur J Cancer 2006; 42: 1671-83.

3. GLOBOCAN 2002 project. International Agency for Research on Cancer. http://www-dep.iarc.fr/ [Consultado el 30 de junio de 2007].

4. International Myeloma Working Group. Criteria for the classification of monoclonal gammopathies, multiple myeloma and related disorders: a report of the International Myeloma Working Group. Br J Haematol 2003; 121: 749-57.

5. Hjorth M, Hellouist L, Holmberg E, Magnusson B, RODJER S, Westin J. Initial versus deferred melphalanprednisone therapy for asymptomatic multiple myeloma stage I a randomized study. Myeloma Group of Western Sweden. Eur J Haematol 1993; 50: 95-102.

6. Riccardi A, Mora O, Tinem C, Valentini D, Brugnatelu $\mathrm{S}$, SPANEDDA R ET AL. Long-term survival of stage I multiple myeloma given chemotherapy just after diagnosis or at progression of the disease: a multicentre randomized study. Cooperative Group of Study and Treatment of Multiple Myeloma. $\mathrm{Br} \mathrm{J}$ Cancer 2000; 82: 1254-60.

7. Dispenzieri A, LaCy M, Greipr P. Multiple Myeloma. En: Greer JP, Foerster J, Lukens JN, Rodgers GM, Paraskevas F, Glader BE (eds). Wintrobe's Clinical Hematology, $11^{\text {th }}$ Edition. Lippincot Williams and Wilkins, 2004; 2583-636.

8. Conte G, Figueroa G, Lois V, Cabrera me, León A, García H, Rojas H. Mieloma múltiple en Chile. Características Clínicas y Sobrevida. Rev Méd Chile 135: 1111-17.

9. Anderson KC, Pazdurn R, Farrell AT. Development of Effective New Treatments for Multiple Myeloma. J Clin Oncol 2005; 23: 7207-11.

10. Kyle RA. Five decades of therapy for multiple myeloma: a paradigm for therapeutic models. Leukemia 2005; 19: 910-2.

11. Conte G, Figueroa G, Lois V, Cabrera Me, León A, García $\mathrm{H}$, Rojas H. Factors of Early Mortality in Patients with Multiple Myeloma (MM). Multicentric Study in Chile (1998-2002). Blood 2006; 108: Abstract \#5007.

12. Augustson BM, Begum G, Dunn J, Barth NJ, Davies F, MORGAN G ET AL. Early mortality after diagnosis of multiple myeloma: Analysis of patients entered onto the United drogas de alta actividad antimieloma. Por otro lado, los pacientes de riesgo estándar obtienen buenas respuestas a los esquemas terapéuticos actuales y se pueden reservar las drogas nuevas para el momento de la recaída o progresión.

Kingdom Medical Research Council Trials between 1980 and 2002 -Medical Research Council Adult Leukaemia Working Party. J Clin Oncol 2005; 23: 9219-26.

13. Stewart AK, Fonseca R. Prognostic and therapeutic significance of myeloma genetics and gene expression profiling. J Clin Oncol 2005; 23: 6339-44.

14. Conte G, Araos D, Figueroa G. Prognostic Factors in Multiple Myeloma. Arch Med Interna 2007; XXIX (Supl 1): S35-S39.

15. Bergsagel PL, Kuehl WM. Molecular pathogenesis and a consequent classification of multiple myeloma. J Clin Oncol 2005; 23: 6333-8.

16. Fonseca R, Barlogie B, Bataile R, et al. Genetics and Cytogenetics of Multiple Myeloma: A Workshop Report. Cancer Res 2004; 64: 1546-58.

17. Gabrea A, Bergsagel PL, Kuehla WM. Distinguishing primary and secondary translocations in multiple myeloma. DNA Repair 2006; 5: 1225-33.

18. Chng WJ, Kumar S, Vanwier S, Ahmann G, Price-Troska T, HENDERSON K ET AL. Molecular dissection of hyperdiploid multiple myeloma by gene expression profiling. Cancer Res 2007; 67: 2982-9.

19. Bergsagel PL, Kuehl WM, Zhan F, Sawyer J, Barlogie B, Shaughnessy J JR. Cyclin D dysregulation: An early and unifying pathogenic event in multiple myeloma. Blood 2005; 106: 296-303.

20. Hanamura I, Stenart JP, Huang Y, Zhan F, Santra M, SAWYER JR ET AL. Frequent gain of chromosome band 1q21 in plasma-cell dyscrasias detected by fluorescence in situ hybridization: incidence increases from MGUS to relapsed myeloma and is related to prognosis and disease progression following tandem stemcell transplantation. Blood 2006; 108: 1724-32.

21. Shou Y, Martelu ML, Gabrea A, Qi Y, Brents LA, RoschKe A ET AL. Diverse karyotypic abnormalities of the c-myc locus associated with c-myc dysregulation and tumor progression in multiple myeloma. Proc Nat Acad Sci NA 2000; 97: 228-33.

22. Tricot G, Sawyer JR, Jagannath S, Desikan KR, Siegel D, NAUCKE $S$ ET AL. Unique role of cytogenetics in the prognosis of patients with myeloma receiving highdose therapy and autotransplants. J Clin Oncol 1997; 15: 2659-66.

23. Fonseca R, Blood E, Rue M, Harrington D, Oken MM, KYLE RA ET AL. Clinical and biologic implications of recurrent genomic aberrations in myeloma. Blood 2003; 101: 4569-75.

24. GerTz MA, Lacy MQ, Dispenzieri A, Greipp PR, Litzow MR, Henderson KJ ET aL. Clinical implications of

A Rtíc U LO

DE REVISIÓN 
$\mathrm{t}(11 ; 14)(\mathrm{q} 13 ; \mathrm{q32}), \mathrm{t}(4 ; 14)(\mathrm{p} 16.3 ; \mathrm{q} 32)$, and $17 \mathrm{p} 13$ in myeloma patients treated with high-dose therapy. Blood 2005; 106: 2837-40.

25. Fonseca R, Harrington D, Oken MM, Dewald GW, Bailey RJ, VAN WIER SA ET AL. Biological and prognostic significance of interphase fluorescence in situ hybridization detection of chromosome 13 abnormalities (delta13) in multiple myeloma: An Eastern Cooperative Oncology Group study. Cancer Res 2002; 62: 715-20.

26. Fonseca R, Blood EA, Oken MM, Kyle RA, Dewald GW, BAILEY RJ ET AL. Myeloma and the t(11;14)(q13;q32): evidence for a biologically defined unique subset of patients. Blood 2002; 99: 3735-41.

27. Avet-Loiseau H, Attal M, Moreau P, Charbonnel C, Garban F, HuUn $C$ ET AL, Genetic abnormalities and survival in multiple myeloma: the experience of the Intergroupe Francophone du Myèlome. Blood 2007; 109: 3489-95.

28. Keats J, Reiman T, Belch A, Pilarski L Ten years and counting: so what do we know about $\mathrm{t}(4 ; 14)(\mathrm{p} 16 ; \mathrm{q} 32)$ multiple myeloma. Leuk Lymphoma 2006; 47: 2289-300.

29. Dring AM, Davies FE, Fenton JA, Roddam PL, Scott K, GonZÁLEZ D ET AL. A global expression-based analysis of the consequences of the $t(4 ; 14)$ translocation in myeloma. Clin Cancer Res 2004; 10: 5692-701.

30. Chang H, Sloan S, Li D, Zhuang L, Yi QL, Chen CI ET AL. The $t(4 ; 14)$ is associated with poor prognosis in myeloma patients undergoing autologous stem cell transplant. Br J Haematol 2004; 125: 64-8.

31. Gutiérrez NC, Castellanos MV, Martín ML, Mateos MV, Hernández JM, Fernández M et al. Prognostic and biological implications of genetic abnormalities in multiple myeloma undergoing autologous stem cell transplantation: $\mathrm{t}(4 ; 14)$ is the most relevant adverse prognostic factor, whereas $\mathrm{RB}$ deletion as a unique abnormality is not associated with adverse prognosis. Leukemia 2007; 21: 143-50.

32. Chesi M, Bergsagel PL, Shonukan O, Martein ML, Brents LA, ChEN T ET AL. Frequent dysregulation of the c-maf proto-oncogene at $16 \mathrm{q} 23$ by translocation to an Ig locus in multiple myeloma. Blood 1998; 91: 4457-63.

33. Hurt EM, Wiestner A, Rosenwald A, Shaffer AL, Campo E, Grogan T ET al. Overexpression of c-maf is a frequent oncogenic event in multiple myeloma that promotes proliferation and pathological interactions with bone marrow stroma. Cancer Cell 2004; 5: 191-9.

34. Chng WJ, Santana-Dávila R, Van Wier SA, Ahmann GJ, Jalal SM, Bergsagel PL et al. Prognostic factors for hyperdiploid-myeloma: effects of chromosome 13 deletions and IgH translocations. Leukemia 2006; 20: 807-13.

35. Chiecchio L, Protheroe RK, Ibrahim AH, Cheung KL, Rudduck C, Dagrada GP et al. Deletion of chromosome 13 detected by conventional cytogenetics is a critical prognostic factor in myeloma. Leukemia 2006; 20: 1610-7.

36. Agnemi L, Bicciato S, Fabris S, Baldini L, Morabito F, INTINI D ET AL. Integrative genomic analysis reveals distinct transcriptional and genetic features associated with chromosome 13 deletion in multiple myeloma. Haematologica 2007; 92: 56-65.

37. Drach J, Ackermann J, Fritz E, Kromer E, Schuster R, GISSLINGER H ET AL. Presence of a p53 gene deletion in patients with multiple myeloma predicts for short survival after conventional-dose chemotherapy. Blood 1998; 92: 802-9.

38. Marzin $Y$, Jamet D, Douet-Guilbert N, Morel F, Le Bris MJ, Morice P ET AL. Chromosome 1 abnormalities in multiple myeloma. Anticancer Res 2006; 26: 953-9.

39. REED S. Ratchets and clocks: the cell cycle, ubiquitylation and protein turnover. Nature Rev Mol Cell Biol 2003; 4: 855-64.

40. Fonseca R, Van Wier SA, Chng WJ, Ketteruing R, Lacy MQ, DisPenZIERI A ET AL. Prognostic value of chromosome 1q21 gain by fluorescent in situ hybridization and increase CKS1B expression in myeloma. Leukemia 2006; 20: 2034-40.

41. Chang H, Qi X, Trieu Y, Xu W, Reader JC, Ning Y et AL. Multiple myeloma patients with CKS1B gene amplification have a shorter progression-free survival post-autologous stem cell transplantation. $\mathrm{Br} \mathrm{J}$ Haematol 2006; 135: 486-91.

42. Lu P, Leong T, Quam L, Biladeau D, Kay NE, GreipP P ET AL. Activating mutations of $\mathrm{N}$-and $\mathrm{K}$-ras in multiple myeloma show different clinical associations: Analysis of the Eastern Cooperative Oncology Group Phase III Trial. Blood 1996; 88: 2699-706.

43. Bezieau S, Devilder MC, Avet-Loiseau H, Melierin MP, Puthier D, Pennarun E et al. High incidence of $\mathrm{N}$ and K-Ras activating mutations in multiple myeloma and primary plasma cell leukemia at diagnosis. Hum Mutat 2001; 18: 212-24.

44. Dispenzieri A, RajKumar SV, Gertz MA, Fonseca R, Lacy MQ, BergSagel PL ET aL. Treatment of newly diagnosed multiple myeloma based on Mayo Stratification of Myeloma and Risk-adapted Therapy (mSMART): consensus statement. Mayo Clin Proc 2007; 82: 323-41.

45. Jagannath S, Richardson PG, Sonneveld P, Schuster MW, Irwin D, StadtMauer EA ET aL. Bortezomib appears to overcome the poor prognosis conferred by chromosome 13 deletion in phase 2 and 3 trials. Leukemia 2007; 21: 151-7.

46. Rosiñol L, Oriol A, Mateos MV, Sureda A, GarcíaSánchez P, GutiérRez N et al. Phase II Pethema Trial of Alternating Bortezomib and Dexamethasone As Induction Regimen Before Autologous Stem-Cell Transplantation in Younger Patients With Multiple Myeloma: Efficacy and Clinical Implications of Tumor Response Kinetics. J Clin Oncol 2007; 25: 4452-8.

47. Palumbo A, Falco P, Corradin P, Falcone A, Di Raimondo F, Giulani N ET al Melphalan, Prednisone, and Lenalidomide Treatment for Newly Diagnosed Myeloma: A Report From the GIMEMA Italian Multiple Myeloma Network. J Clin Oncol 2007; 15: 4459-65. 